\title{
Research on Lighting Design Method of Children's Ward
}

\author{
Li Wanling ${ }^{1, a}$, Ren Shaohui ${ }^{2, b^{*}}$

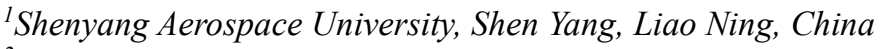 \\ ${ }^{2}$ Shenyang Aerospace University, Associate professor, Shen Yang, Liao Ning, China \\ a*790104381@qq.com \\ b*728000158@qq.com
}

\begin{abstract}
Light is a way of energy transmission, which has a great impact on children's visual health. Lighting environment is one of the important factors affecting the comfort of wards. Therefore, the introduction of lighting design in the interior design of wards has an important impact on the physical rehabilitation of children. Based on the actual investigation of ward space and the needs of ward lighting service subjects, this paper analyzes the design requirements of healthy lighting environment in children's wards and puts forward the application methods in the actual lighting planning process. The purpose of this study is to provide design strategies for the construction and development of medical space, and to provide a healthy, comfortable and safe ward lighting environment for hospitalized children.
\end{abstract}

Keywords: Ward, lighting design, children

\section{儿童病房照明设计方法研究}

\author{
李婉翎 $1, \mathrm{a}$ 任绍辉 $2, \mathrm{~b}^{*}$ \\ ${ }^{1}$ 沈阳航空航天大学工业设计工程系研究生, 沈阳, 辽宁, 中国 \\ 2 沈阳航空航天大学设计艺术学院副教授, 沈阳, 辽宁, 中国 \\ a*790104381@qq.com \\ b*728000158@qq.com
}

\section{摘要}

光是能量的一种传播方式，对儿童的视觉健康有很大影响，照明环境是影响病房舒适度的重要因素之一，所以 在病房室内设计中引入照明设计对患儿的身体康复有重要的影响。本文从对病房空间的实际调研和病房照明服 务主体人群的需求出发, 分析儿童病房健康照明环境的设计需求, 提出在实际灯光计过程中的运用方法。研究 目的是希望为医疗空间的建设发展提供设计策略, 为住院儿童设计一个健康、舒适、安全的病房照明环境。

关键词: 病房, 照明设计, 儿童

\section{1. 前言}

近些年新改建后医院的功能在不断升级变化，医 疗设备愈加先进, 病房是医院建筑中最常见的一类空 间, 具有生活、治疗、康复等多重功能, 病房的室内 设计也越来越符合人的审美取向。但病房在照明方式 上显得过于注重功能性, 缺乏对患者心理和情感取向 的关注。另一方面, 在新医疗改革和三孩政策的推动 下, 儿童健康问题越来越受到国家和社会的关注和重
视。

灯光设计是室内设计的重要一环, 而良好的病房 环境对病人的休息疗养起到一定的积极作用。目前, 除了某些特殊疾病之外, 并没有专门针对儿童来制定 相关的照明设计准则。照明设计师可以通过一些照明 技术手段来辅助缓解儿童住院时期不安的情绪。

目前我国制定了《建筑照明设计标准 (GB50034 -2013), 对医疗建筑室内照明做了规定标准。国际照 
明委员会（CIE）《室内工作场所的照明》定了医院 不同区域参考平面上的维持照度、统一眩光等级限 制、最低显色指数及特殊情况建议, 包括病房里不同 作业时的具体要求 $[1,2]$ 。对室内照明而言, “符合 照度标准” 的设计并不是一个最佳设计方案; 一个优 秀的儿童病房照明设计应该考虑到灯光可能会对患 儿、医护人员、患儿家属带来生理与情绪的正面影响。

\section{2. 照明设计在儿童病房空间中的重要性}

\section{1 现状分析}

作者对成都一处大型医院进行了现场调研, 使用 SPIC-300AW 照度仪进行现场数据采集, 统计分析了 当前环境照明现状主要问题。并收集了 20 份 8-15 岁 住院儿童、28 份陪护家属、5 位医护人员对当前病房 照明环境的主观调查问卷。

成都市某大型医院两人间的儿童病房护理单元， 病床上方天花安装有吸顶灯, 提供主要均匀下照光线 的照射, 照亮病床及周边环境; 病床床头线形灯 45 度斜出光方向光线局部重点照明; 病房通行区域天花 上方每间隔 1.5 米设置一个下照筒灯, 共三个。卫生 间门口侧壁拐角处有地脚夜灯设置, 方便病患起夜。 病房整体照度、色温、照明方式都比较单一, 整体光 环境无层次感。卫生间洗手台镜前灯处、淋浴间处天 花各一盏下照筒灯。在所有照明灯具全开模式下, 通 过照度仪测试得到病床面照度 575. 31x, 色温 5312k; 通行区域地面照度 $135.01 x$, 色温 5000k; 卫生间地面 照度为 $162.51 x$, 色温 $5062 \mathrm{k}$; 地脚灯 $541 \mathrm{x}$, 色温 $3000 \mathrm{k}$ 。总结成以下几点:

1. 照度不均, 灯具配光不合理; 设计师不仅要考 虑水平面的照度是否达标, 也要考虑垂直面的照度指 标是否合格。

2. 眩光和阴影问题严重; 通过问卷调查和谈话交 流得知患儿躺卧时的主观感受是明显眩光。现有的照 明方式, 忽视了儿童的生理特点。儿童缺乏自我保护 意识, 只能被动接受所处的光环境; 但是儿童敏感的 视神经发育更需要设计者考虑到眩光控制。

3. 色温偏高, 病房整体色温在 4500-5000k, 最 高达到 $6100 \mathrm{k}$, 儿童在活动时, 会有冷酷的感受, 缺 乏温馨感。

4. 照明方式单一, 照明与空间需求对应欠缺。儿 童病房需要明确针对儿童患者的主体, 在空间照明设 计中要考虑儿童患者的喜好和感受。照明不能根据需 求灵活调整, 不能从多个角度满足病房工作人员的需 求。

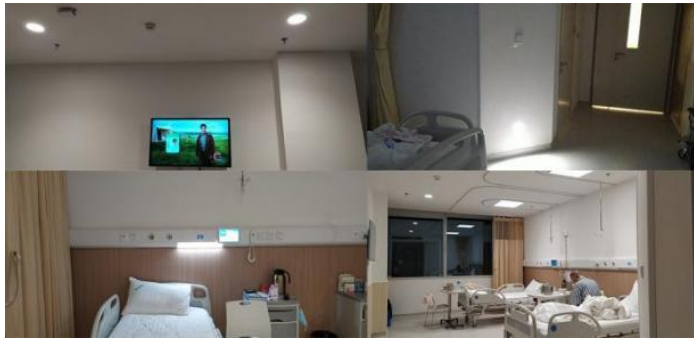

图 1 成都京东方医院病房实际情况

从本次调研中可以看到我国内地新改建医院的 儿童病房环境现况较以前已有很大提升。但是在成人 与儿童的尺度上并没有太大功能区分; 在设计过程中 往往只重视解决基本亮度、照度、色温、灯具显色性 等照明硬性指标。

\section{2 使用人群需求分析}

儿童病房的主要使用人群有三类: 患儿、医护人 员和陪护家属, 其中最重要的是患儿。

通过调研沟通我们得到来自孩子和家人的一些 行为信息。因为病情原因, 患儿大多时间处于躺卧状 态, 所以对天花灯具的选择尤为重要。儿童对医院的 恐惧和焦虑比成人更重, 所以他们具有强烈的情感需 求。儿童因年龄小, 情感表露直率、单纯, 表现为恐惧、 胆怯、悲伤等; 他们更加希望拥有使其感到平静熟悉 的环境, 就好像处在幼儿园、家里的氛围中。所以病 房的色彩使用应该尽量活泼, 增加童趣元素, 营造轻 松的修养康复氛围, 吸引患儿注意力, 减轻住院恐惧。

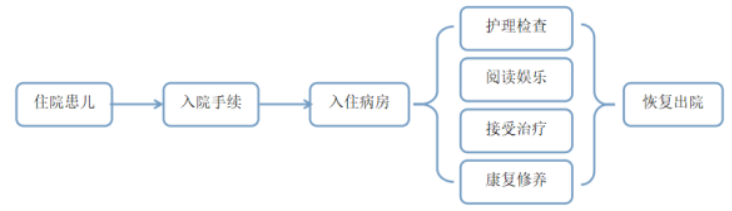

图 2 住院患儿行为动线

另一方面, 设计工作者还要考虑到陪护家属的心 理状态和情绪需求, 更利用光的要素来排解其消极情 绪, 增强家长信心的同时也使得孩子能尽早的开始积 极治疗。在对儿童病房照明设计工作中, 考虑到光的 的引导作用、功能作用。合理运用设计使患儿以及家 属获得轻松的住院体验, 让陪护家属的这一灯光体验 带入记忆中, 人们对医院的印象就会逐渐变好。

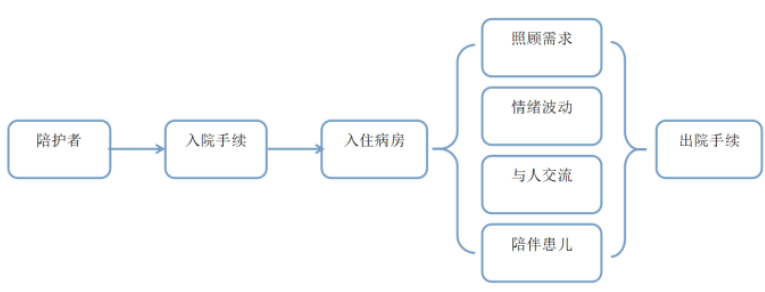

图 3 陪护家属行为动线

最后是医护人员。医护人员在病房内从事各类视 觉作业, 主要包括: 观察了解病患的基本身体状况 和体征; 查看患儿抽血输液输氧情况; 记录病情变化。 


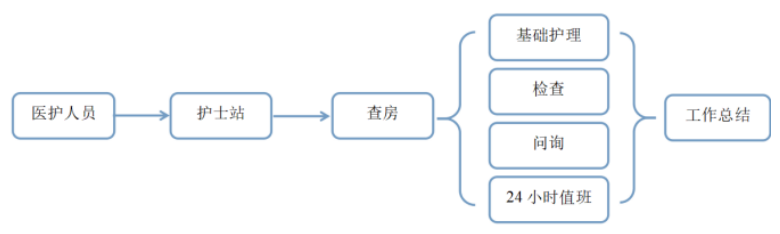

图 4 医护人员行为动线

\section{3. 照明设计在儿童病房空间中的应用原则}

\section{1 视觉健康原则}

在医疗建筑灯光设计中, 要处理好照明方式、色 温、照度等重要因素, 关注儿童的身心健康和正常发 育。

儿童的视觉健康人类的情绪是在特定照明环境 中不同情感的具体表现。在病房照明环境中, 自然光 可以加强物体的立体感, 使事物和景观更生动; 视觉 感知产生的情绪可以帮助人类感受到空间内的照明 系统所共同塑造的环境氛围, 并且这一环境对人类自 身的情感及情绪也会产生影响 [3]。

患儿在病房中最长的行为是卧床, 所以在天花板 灯具选择时一是灯具安装位置, 避免安装在病床正上 方, 二是提高照明质量, 使用间接照明方式或者对灯 具进行防眩处理, 添加防眩格栅。并且选择多组合的 照明方式, 满足不同时间段和不同行为状态的灯光需 求。

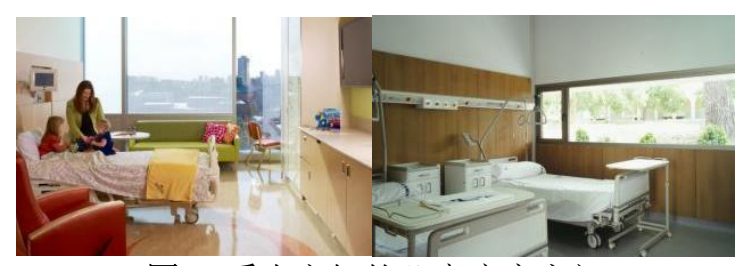

图 5 采光良好的儿童病房空间

\section{2以人为本原则}

儿童是一个特殊的群体, 心智相对于成人而言更 加易处于不成熟脆弱阶段, 住院患儿容易处于焦虑、 恐惧的状态, 在照明设计中充分考虑各个方面的细 节, 尽量减少患儿的不安情绪 [4]。考虑到孩子视觉 敏感度比成人要高, 所以要合理控制亮度和色温, 避 免过亮或过低伤害孩子的视觉健康。

合理选择照明方式。照明方式分为直接照明、半 直接照明、间接照明、半间接照明、漫射照明 5 类。 在医疗空间的室内照明设计中, 采用混合照明的方 式, 光源在被照明对象上, 光量弱、光线柔和、无眩 光和明显暗影, 避免直射患儿的眼睛, 营造安静、祥 和的空间环境。除了雅观、安全因素, 还要合理分布 光源、光线照射方向和强弱 [5]。在人 0-14 岁的过程 中, 身心成长具有明显的独特性和过渡性, 因此, 儿 童空间照明应遵循其儿童的生理特征和心理规律。

\section{3 绿色安全原则}

儿童空间照明设计首选 LED 新型灯具。绿色照明 设计理念是指在空间中, 通过科学的照明理念设计, 运用先进的科学技术和方法, 采用节能、环保、高效、 安全的新型照明产品, 从而给人们创造出一个舒适、 温馨的康复病房环境, 并且体现现代科学技术给我们 生活带来的新型照明设计 [6]。遵循绿色照明设计理 念, 另一个必须考虑到的是安全性。安装位置易被患 儿触碰到的灯具应为封闭式，防护性高。

良好的采光可以缓解患者的情绪, 提高恢复率, 缩短恢复期, 前提是患者在住院病房的视野较好。自 然光可提供了良好的室内高度, 减少了白天开灯时 间, 减少了白天照明的能耗。

\section{4 亲情关怀原则}

家属作为陪伴患儿必不可少的人群, 他们的不良 视觉感受影响生理心理、精神再反映到患儿身上, 不 利于儿童疾病的康复。所以在设计中要考虑到人这一 主体的感受, 让患儿、陪护家属、医护人员三者的要 求达成统一, 也是儿童病房照明设计原则中的一项重 要内容。

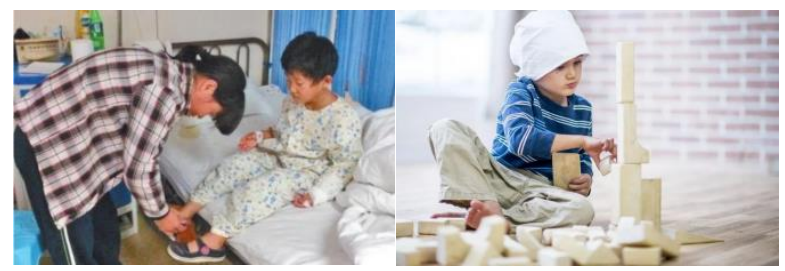

图 6 儿童病患情绪

儿童对形状和色彩特别敏感, 视觉是儿童获取外 部信息的主要来源。随着照明技术的发展和照明形式 的多样化, 照明设计师和照明厂商可以在灯具选择和 LED 照明组合的形式上探索照明的亲密性。

\section{5 节律引导原则}

因为没有改善灯光环境的病房很长一段时间, 节 奏紊乱带来的相移的生物钟, 影响患儿的活动、进食、 睡眠和唤醒甚至是学习和记忆能力、情绪和其他明显 的昼夜节律波动。

除了一般照明设计主要关注可见性问题, 如减少 眩光和阴影、显色性、安全性和美观性等, 节律调节 引导进行照明设计时, 会涉及更多不同的照明设计目 标。儿童在视觉搜索、视觉空间构建、视觉区分等方 面具有一定优势。可以通过节律灯光照明利用不同的 亮度调光模式, 在不同的季节和全天不同的时段遵循 人与自然的节律。节律性体现在尺度、材质和光所传 达的信息。光照节律影响、亮度和色温可以跟随不同 时间段、医护人员检查、儿童活动的多样性而改变。 照明通过不同的亮度调光模式，在不同的模式和全天 不同的时段遵循人与自然的节律。节律性体现在尺 度、材质和光所传达的信息。 


\section{4. 照明设计在儿童病房空间中的设计方法}

医护人员在对患儿进行身体护理时, 对照度有着 较高需求, 9001x 满足检查照明照度, 在日常生活中 1001x 则可以满足患者所需要的一般照明需求, 而深 夜病房内的少量活动照度在 5-101x。考虑儿童视觉 灵敏度较高, 在深夜亮度控制和日常活动时, 按照场 景不同，提供较低的照度和日常活动时较高照度。

\section{一、休息疗养场景模式}

在休息疗养场景模式中，病人是空间中的主体， 一切照明行为应从病人的角度展开, 患儿的生理节 律、舒适感、与安全性是要首先考虑的, 所以在满足 护理人员需求的同时也要满足患儿的需求。感受到心 理的慰藉。儿童的视力脆弱易受刺激, 照明需要柔和 的光线。因此, 病房照明应采用直线型灯具或反光灯 具。灯应远离床, 以免使卧床的儿童眼花。

\section{二、护理检查场景模式}

在护理检查场景模式中, 要立足于满足医生需求 的照明设计模式。医护人员需要密切关注患者病情, 掌握其动态变化情况, 照度为 $10001 x$, 色温为 $5700 \mathrm{k}$, 采用高照度高色温的照明模式, 偏冷色光不仅可以让 医生在检查过程聚精会神, 满足其诊断、护理等工作 需要, 也可以给患者一种镇定的感觉。床头安装医疗 设备带和床头灯带, 既不影响其他病人休息, 医生检 查时也可用床头灯做重点照明。

\section{三、夜间活动场景模式}

病房中的部分灯具采用可控 $2500 \mathrm{k}-5000 \mathrm{k}$ 色温 亮度的 LED 灯具, 根据患者的活动状态设置不同模 式, 并且夜间可以单独控制开关。另外, 病房内设置 照明的光线需与人受光源的向导性这一知觉模式相 符, 在凌晨情况下, 将休息护理照明模式中线性间接 照明光源以低照度低色温的模式开启, 在对环境进行 照亮的情况下, 利用人的视觉导向性使人能够正确判 定空间的方向, 地脚灯选用感应模式, 人靠近的时候 亮, 人远时灭。如图 7 所示, 对人在空间中的行走起 引导指向作用。

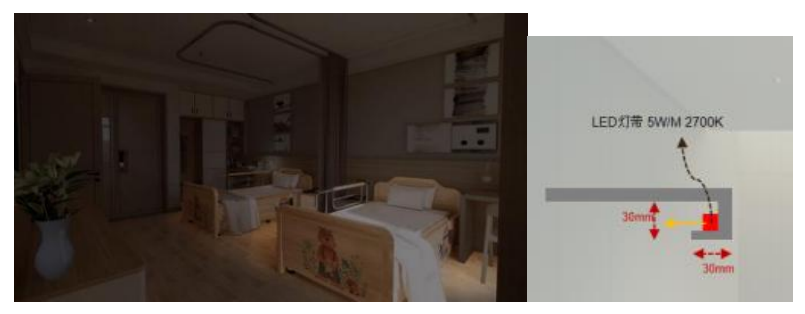

图 7 夜间病房照明方式效果及节点分析

\section{5. 结论}

病房过度照明将会导致病患的负面情绪, 在现阶 段儿童病房照明环境存在的问题、空间环境条件及照 明主体人群的视觉、心理、行为需求的基础上提出设 计原则和设计策略并设定三种不同的照明场景模式:
休息疗养场景模式、护理检查场景模式和夜间活动场 景模式的设计应用来满足患儿、家属、医护人员需求 及照明功能需求。在未来的儿童病房照明尺度上需要 更多考虑到儿童的生理节律、行为动线。以及在设计 中适当增加一些装饰性的灯光营造一个轻松的灯光 小环境, 减少儿童住院的负面情绪。

\section{REFERENCES}

[1] CIE158: Ocular lighting effects on human physiology and behavior. Publication CIE, 2004.

[2] Xu Junli, Luoxi Hao, etc. Evidence-based Research and Practice on Healthy Light Environment in wards, 2019.

[3] Srazali Aripin. Healing architecture: A study of daylight in public hospital designs in Malaysia. 2010 .

[4] Safaa Alzubaidi, Prashant Kumar Soori. Energy Efficient Lighting System Design for Hospitals agnostic and Treatment Room - A Case Study. Journal of Light and Visual Environment, 2012.

[5] Cai, W., J. Yue, Q. Dai, etc. The impact of room surface reflectance corneal illuminance and rule-of-thumb equations for circadian lighting sign [J]. Building and Environment, 2018.

[6] Yang He, Liu Chang, etc. Application of artistic expression of lighting design in homestay,2018. 